\title{
The performance of gradient alloy quantum dots in cell labeling
}

Stefaan J. Soenen ${ }^{\mathrm{a},{ }^{*}}$, Bella B. Manshian ${ }^{\mathrm{a}}$, Uwe Himmelreich ${ }^{\mathrm{a}}$, Jo Demeester ${ }^{\mathrm{b}}$,

Kevin Braeckmans ${ }^{\mathrm{b}, \mathrm{c}}$, Stefaan C. De Smedt ${ }^{\mathrm{b}, *}$

${ }^{a}$ MoSAIC/Biomedical NMR Unit, Department of Medicine, Catholic University of Leuven, B3000

Leuven, Belgium

${ }^{\mathrm{b}}$ Lab General Biochemistry and Physical Pharmacy, Faculty of Pharmaceutical Sciences, University of Gent, B9000 Gent, Belgium

${ }^{\mathrm{c}}$ Center for Nano- and Biophotonics, Faculty of Pharmaceutical Sciences, University of Gent, B9000 Gent, Belgium

Corresponding author: $\quad$ Stefaan J. Soenen: $\underline{\text { Stefaan.Soenen@med.kuleuven.be }}$

Stefaan C. De Smedt: $\underline{\text { Stefaan.DeSmedt@ugent.be }}$ 


\begin{abstract}
The interest in using quantum dots (QDots) as highly fluorescent and photostable nanoparticles in biomedicine is vastly increasing. One major hurdle that slows down the (pre)clinical translation of QDots is their potential toxicity. Several strategies have been employed to optimize common coreshell QDots, such as the use of gradient alloy (GA)-QDots. These particles no longer have a sizedependent emission wavelength, but the emission rather depends on the chemical composition of the gradient layer. Therefore, particles of identical sizes but with emission maxima spanning the entire visible spectrum can be generated. In the present study, two types of GA-QDots are studied with respect to their cytotoxicity and cellular uptake. A multiparametric cytotoxicity approach reveals concentration-dependent effects on cell viability, oxidative stress, cell morphology and cell functionality (stem cell differentiation and neurite outgrowth), where the particles are very robust against environmentally-induced breakdown. Non-toxic concentrations are defined and compared to common core-shell QDots analyzed under identical conditions. Additionally, this value is translated into a functional value by analyzing the potential of the particles for cell visualization. Interestingly, these particles result in clear endosomal localization, where different particles result in identical intracellular distributions. This is in contrast with CdTe QDots with the same surface coating, which resulted in clearly distinct intracellular distributions as a result of differences in nanoparticle diameter. The GA-QDots are therefore ideal platforms for cell labeling studies given their high brightness, low cytotoxicity and identical sizes, resulting in highly similar intracellular particle distributions which offer a lot of potential for optimizing drug delivery strategies.
\end{abstract}




\section{Introduction}

In the field of nanomedical research, quantum dots (QDots) take an important place as powerful probes for fluorescence imaging [1-3]. QDots are colloidal nanocrystals with a core diameter that ranges between 1 and $10 \mathrm{~nm}$ and that are built up of semiconductor materials, typically combinations of groups 12 and 16 (e.g. CdTe, CdSe, ZnS). QDots possess excellent fluorescent properties such as a high photostability, very high brightness and very narrowand sizetunable emission spectra [4]. These properties enable a broad range of applications such as multiplexed imaging of fixed cells, tissues and even clinical tissue samples, enhancing the predictability of outcome of cancer progression at more early stages [5,6]. Owing to their high photostability and brightness, QDots are also extensively used for kinetic single molecule studies on cellular proteins such as kinesin or individual glycine receptors, providing detailed information on their intracellular mobility and processing that was not previously possible using organic fluorophores $[7,8]$.

In biomedical research, $\mathrm{Cd} 2+$-containing QDots are most commonly used as they possess superb optical properties and synthesis protocols have been well-described [9]. Both core-only (e.g. CdTe) or core-shell QDots exist (e.g. CdSe/ZnS) where the shell layer passivates the core, enhancing its optical properties and typically rendering the QDots more compatible with a biological environment by shielding the $\mathrm{Cd} 2 \mathrm{p}$-containing core from the environment [10]. Although these $\mathrm{Cd} 2+$-containing QDots have proven to have a lot of potential for a broad variety of biomedical applications, they currently remain limited to proof-of-concept studies. One major hurdle concerning the full exploitation of $\mathrm{Cd} 2+-$ containing QDots is their potential toxicity, derived from the high toxicity of free $\mathrm{Cd} 2+$ ions [11]. Although the gradual release of toxic ions can be exploited for biomedical purposes such as cancer therapy [12], it may also inadvertently affect healthy cells as long as this process remains only partially understood. A lot of attention has been put in determining the potential toxicity of $\mathrm{Cd} 2+-$ containing QDots, but thus far, the safety of these NPs remains questionable due to several intrinsic difficulties that are associated with assessing NP safety: 1) the wide number of variations in QDot parameters (purity of the NPs, colloidal stability, chemical composition,size, core-only or core-shell, nature and thickness of the shell layer, type of coating ...) [13], 2) the nature of the experiments performed (e.g. differences in cell types, media composition, experimental procedures such as incubation times, concentrations used...) [14], 3) discrepancies between in vitro and in vivo data due to intrinsic differences in experimental setups (effect of agglomeration and sedimentation, effect of continuous flow versus static model) $[15,16]$. All these parameters influence the results obtained and therefore harden any comparison of toxicity data, generating a lot of confusion regarding the potential toxicity or safety of Cd2+-containing QDots. Although a recent pilot study indicated that these NPs do not cause any acute toxicity in non-human primates [17], long-term effects were not looked into and need to be considered carefully [18]. In vitro data often show high toxicity levels, which are to a large extent caused by the exposure of the QDots to their immediate biological (and degradative) 
environment, being cellular endo- or lyso-somes and their behavior in this complex biological environment, which results in leaching of free $\mathrm{Cd} 2+$ ions and thereby inducing toxicity [19-23].

To overcome these toxicity-related issues, a lot of focus has been put into optimizing QDot design to generate NPs with increased biocompatibility. One possibility lies in the generation of $\mathrm{Cd} 2+$-free QDots, but thus far, the synthesis of these QDots remains to be further optimized and a lot of effort is being put into increasing their optophysical properties to be in the same line as the $\mathrm{Cd} 2+$-containing ones [24,25]. The coating of the QDots can also be modified, where double polymer-silica coatings have been shown to enhance the resilience of the QDots against environmental degradation of the QDots [26]. A third option lies in modifying the chemical composition of the QDots, where the use of either core/shell/shell QDots or alternatively, so-called gradient alloy QDots have been put forward as powerful alternatives with excellent opticophysical properties [27-29]. Gradient alloy QDots consist out of a gradient internal structure (e.g. cadmium zinc selenide) which in contrast to particles with a homogeneous structure enables to achieve a fine tuning of the optical properties of the QDots without having to change particle size [30]. By modifying this gradient, gradient alloy QDots of the same size have been generated that can emit in the entire visible range [31]. Gradient alloy QDots also exhibit excellent opticophysical properties, including high quantum yields and photostability and resistance against surface etching [29]. Of specific interest, gradient alloy QDots have also, for the first time, been found to be able to be tuned into non-blinking QDots, exhibiting continuous photoluminescence [32]. This property renders them highly valuable for a multitude of applications, such as the continuous follow-up of single molecules in live cells [32,33].

Given these optimal features, gradient alloy QDots appear to be highly valuable alternatives to common coreeshell QDots, but their potential toxicity remains to be investigated thoroughly. To this end, the present work focuses on evaluating the cytotoxicity of two types of gradient alloy QDots $(\mathrm{CdZnSe} / \mathrm{ZnS})$ with emission maxima of 508 and $600 \mathrm{~nm}$, respectively. A multiparametric analysis of particle toxicity is then performed according to previously established methods, in which a sequential analysis is made of QDot uptake, cytotoxicity, influence of endosomal $\mathrm{pH}$, oxidative stress, cell morphology and cell functionality using a panel of three different cell types [14]. In the end, a concentration will be derived at which no effect can be observed for any of the parameters described above. As determining NP concentrations is a tedious task and prone to errors [13], this value does not allow for a straightforward comparison with other materials tested previously under identical conditions. Therefore, the functionality of the QDots will also be evaluated in terms of their ability to track live cells when labeled at non-toxic concentrations and this value can then be used to compare to other particles with the same functionality that have been tested previously, including different types of core-shell QDots [19,22]. Additionally, the effect of identical particle sizes but different emission spectra will also be tested in terms of the intracellular localization of the particles. In contrast to most QDot toxicity studies to date, the present study therefore addresses particle functionality at non-toxic 
levels and compares the data obtained to the data obtained for other types of QDot tested under identical conditions. The multiparametric methodology therefore enables to define the mechanisms underlying QDot toxicity and furthermore allows to compare the functionality of all different types of QDots previously tested under identical conditions, making it a very powerful technique. 


\section{Materials and Methods}

\subsection{Nanoparticles}

Two types of gradient alloy QDots were purchased from Mesolight LLC (Little Rock, Arkansas, USA). Both QDots contained a $\mathrm{Cd}_{\mathrm{x}} \mathrm{Zn}_{1-\mathrm{x}} \mathrm{Se}$ core and a $\mathrm{ZnS}$ shell. The differences in emission spectra are due to differences in the exact composition of core. The particles were provided as QDots with emission maxima at 508 and $600 \mathrm{~nm}$. To ensure colloidal stability in an aqueous environment, the particles were both coated with 3-mercaptopropionic acid, resulting in a net negative charge for electrostatic repulsion. The particles were provided as $9 \mu \mathrm{M}$ QDot stock solutions in alkaline $\mathrm{H} 2 \mathrm{O}, \mathrm{pH}$ 11. For the effect of size on intracellular QDot distribution, two types of CdTe QDots (2.6 and $6.4 \mathrm{~nm}$ diameter, with emission maxima of 510 and $630 \mathrm{~nm}$, respectively) were purchased from Plasmachem $\mathrm{GmbH}$ (Germany). These QDots were also coated with 3-mercaptopropionic acid, therefore having similar surface properties as the GA-QDots.

\subsection{Nanoparticle characterization}

\subsubsection{Size determination by transmission electron microscopy}

The size of the QDots was determined by transmission electron microscopy (TEM) using a Cs corrected JEOL 2200 FS microscope operating at $200 \mathrm{kV}$.

\subsubsection{Elemental analysis}

The chemical composition of the QDots was determined by energy dispersive Xray spectroscopy (EDX) using the same microscope as for the TEM observations.

\subsubsection{Emission spectra}

Emission spectra of the QDots were recorded using a Quantifluor fluorometer (Promega, Belgium) with an excitation wavelength of $340 \mathrm{~nm}$.

\subsubsection{Dynamic light scattering and electrophoretic mobility measurements}

The hydrodynamic diameter and $\zeta$-potential of both types of QDots were measured using a Nanosizer instrument (Malvern,Worcestershire, UK). To this end, the QDots were diluted (1/500) in phosphate buffered saline (PBS: $10 \mathrm{mM}$; pH 7.4) after which the measurements were performed (12 cycles/run) in triplicate.

\subsubsection{QDot sterility tests}


Both types of QDots were provided as sterile stock suspensions. To rule out the effect of any biological contaminants such as endotoxins, the common endpoint chromogenic QCL-1000® LAL assay (Lonza, Verviers, Belgium) was performed according to the manufacturer's instructions.

\subsection{QDot-cell interaction studies}

Following cellular exposure to the different QDots at various concentrations, the following parameters were evaluated: confocal microscopy of intracellular QDot localization and quantification of QDot clusters and quantitative levels of cell-associated QDots by fluorescence intensity measurements of lysed cells. The effect of the surrounding $\mathrm{pH}$ on QDot fluorescence intensity, and $\mathrm{Cd} 2+$ release was determined spectrophotometrically. Cell viability was evaluated in proliferating and non-proliferating cells (to determine the effect of intracellular QDot degradation on cellular health) using an Alamar blue assay. Oxidative stress was evaluated spectrofluorometrically using CellROX Green. Cell functionality was evaluated by fluorescence microscopy of HUVEC cells stained for actin and $\alpha$ tubulin (cell spreading), C17.2 cells stained for TuJ-1 (neuronal differentiation), and PC12 cells stained for $\alpha$-tubulin (nerve growth factor-stimulated neurite outgrowth). Intracellular QDot distribution and endosomal localization of green and red-emitting GA-QDots and CdTe QDots were evaluated by confocal microscopy. Detailed experimental methodologies can be found in the Supporting information that accompanies this manuscript.

\subsection{Statistical analysis}

All data are expressed as mean \pm SEM unless indicated otherwise and analyzed using one-way analysis of variance (ANOVA). Comparisons between particle-treated groups and untreated control groups were analyzed using the Dunnett post-hoc analysis method. In all cases, the degree of significance is indicated when appropriate $(*: \mathrm{p}<0.05 ; * * \mathrm{p}<0.01 ; * * * \mathrm{p}<0.001)$. 


\section{Results and Discussion}

\subsection{Nanoparticle characterization}

The QDots (QDot500 and QDot600) were received as sterile colloidal suspensions where the particles were provided at a $9 \mu \mathrm{M}$ stock concentration in alkaline $\mathrm{H} 2 \mathrm{O}, \mathrm{pH} 11$. The presence of any endotoxins was evaluated using the common endpoint chromogenic QCL-1000® LAL assay, which did not show any endotoxins present in the QDot stock suspensions (data not shown). The QDots used in the present study had different emission maxima, being 508 and 600 nm, respectively (Supporting Fig. S1) and photoluminescence quantum yields of 41 and $90 \%$. These values are in line with earlier reports on gradient alloy QDots, reaching very high PLQY compared to more classical coreeshell structured QDots [30]. The two types of QDots were therefore further defined as QDot500 and QDot600. The QDots used in the present study consist of a gradient layer of $\mathrm{Cd}_{\mathrm{x}} \mathrm{Zn}_{1-\mathrm{x}} \mathrm{Se}$ as core surrounded by a $\mathrm{ZnS}$ outer shell. Inside the core, the ratio of $\mathrm{Cd}$ and $\mathrm{Zn}$ varies, as demonstrated by elemental analysis (Table 1, Supporting Fig. S2) resulting in 2 QDot types of similar size (5.2 $\pm 0.8 \mathrm{~nm}$ for QDot500 and $4.8 \pm 0.7 \mathrm{~nm}$ for QDot600) as determined by transmission electron microscopy (Supporting Fig. S2). The difference in emission maxima therefore is linked to differences in the chemical composition of the QDots rather than differences in their size. The particles were both coated with short ligands, being 3-mercaptopropionic acid (MPA), which resulted in QDots with a negative surface charge of -34 $\pm 3 \mathrm{mV}$ (QDot500) and $-33 \pm 2 \mathrm{mV}$ (QDot600) as determined by electrophoretic mobility measurements. The particles were found to be rather stable in their alkaline environment, but upon transferring them to cell culture media, agglomerates were formed that remained stable for up to at least $24 \mathrm{~h}$. The hydrodynamic diameter of both particles after $24 \mathrm{~h}$ exposure to cell culture media equals $117 \pm 16 \mathrm{~nm}$ (QDot500) and $117 \pm 14 \mathrm{~nm}$ (QDot600) (Supporting Fig. S3).

\subsection{Effect of endosomal pH on QDot fluorescence}

To evaluate how useful these QDots are for biological applications such as cell labeling, it is essential to assess the effect of the different environments to which the QDots will be exposed during its cellular internalization by the cell. Small NPs such as QDots are typically endocytosed by cultured cells [34], which results in their enclosure in endosomes and in a later phase, lysosomes, which are degradative cellular organelles that have a low $\mathrm{pH}$ (5.5 for endosomes, 4.5 for lysosomes). This low pH can affect the QDots by degrading their organic surface coating [35], following by acid etching of the QDot surface, resulting in a reduced PLQY and release of $\mathrm{Zn} 2+$ and $\mathrm{Cd} 2+$ ions $[19,22]$. The intracellular release of these ions has been shown to be an important mediator in QDot toxicity [36,37]. To evaluate how stable these gradient alloy QDots are against $\mathrm{pH}$-dependent degradation, the effect of physiologically relevant $\mathrm{pH}$ levels on QDot properties (PLQY) was investigated, using previously established protocols as detailed in the Supporting Materials and methods. 
When the particles were exposed to citrate-containing buffer systems of $\mathrm{pH} 5.5$ and 4.5 , a clear reduction in fluorescence intensity of both types of QDots can be seen (Fig. 1). Interestingly, the loss in fluorescence intensity is far lower than previously observed for several types of $\mathrm{CdSe} / \mathrm{ZnS}$ coreeshell QDots studied under identical conditions [19]. For the latter core-shell QDots after 5 days at $\mathrm{pH} 4.5$, fluorescence intensity had dropped to approximately $40 \%$. Together, these data show that the gradient alloy QDots studied in the present work appear to be more stable against pH-mediated degradation. This may be explained by the fact that the crystal structure of the alloy layer is more robust and less prone to environmentally induced defects such as oxidation, as shown for different types of GA-QDots that resulted in bright QDots with high PLQY, even after transfer from the organic to aqueous solvent [29]. The high brightness and $\mathrm{pH}$-stability of the gradient alloy QDots suggest that these particles have a high potential for biological applications.

\subsection{Cellular uptake of gradient alloy QDots}

As the uptake mechanism and final intracellular localization of NPs may differ depending on the size of the NPs and their specific surface chemistry [34], the cellular uptake of the QDots was investigated. In the present work, three different cell types are used (murine C17.2 neural progenitor cells, primary human umbilical vein endothelial cells (HUVEC) and rat PC12 pheochromocytoma cells) that have previously been shown to have a high potential in cell therapy and which are excellent models for different tissue types [38,39]. Cells that were transiently expressing lysosomal associated membrane protein 1 (Lamp1) fused with a fluorescent protein (green or red) as a marker for late endosomal and lysosomal compartments were exposed to the QDots at concentrations up to $50 \mathrm{nM}$ for $24 \mathrm{~h}$. Cells were then analyzed by confocal microscopy, where the colocalization of the QDots and the lysosomal marker was analyzed (Fig. 2A). The data reveal a clear colocalization of either QDot500 or QDot600 with the fluorescent lysosomes, indicating that both types of QDots were taken up by the cells through the process of endocytosis, as is the case for most types of NPs [34]. The gradient alloy QDots thus appear to finally reside in the lysosomal compartment of the cell, where they will be exposed to the low $\mathrm{pH}$ as described above.

For toxicological purposes, it is also essential to quantify the number of NPs per cell as the total number of cell-associated NPs is often more important than the total number of NPs initially administered to the cells [22]. Particle toxicity can sometimes be reduced by applying an alternative coating to the NPs [40], which often results in a reduced cytotoxicity merely by decreasing cellular NP uptake. Therefore, in order to investigate NP toxicity, it is essential to correlate NP uptake levels with cellular toxicity. Additionally, for the purpose of cell labeling, the QDots used in the present study must exert a low toxicity while reaching high levels of intracellular NPs. Based on fluorescence images taken of cells exposed to the QDots for $24 \mathrm{~h}$, the number of QDot-positive spots is determined

(Fig. 2B, Supporting Fig. S4). This value indicates how well the QDots can be used to visualize the 
entire cell by fluorescence microscopy. However, for toxicity purposes, the number of QDot-positive vesicles is less interesting as QDots typically cluster together within the small luminal space of the endosomes and the number of QDots per endosome can vary a lot. Quantifying the total number of QDots per cell based on their fluorescence is not very straightforward, as the fluorescence intensity level of the QDots will be affected by the $\mathrm{pH}$ and ionic strength of their environment as well as by their state of agglomeration [41]. Therefore, the number of QDots per cell were quantitated using previously established protocols, in which labeled cells are slowly lysed in alkaline conditions, after which the number of QDots can be quantified more accurately [19]. Fig. 2C (Supporting Fig. S4) shows that both types of QDots have similar uptake levels, as expected by their similar size and identical surface chemistry.

\subsection{Acute and long-term toxicity and oxidative stress}

As the cellular uptake levels of either type of QDotwas sufficient to efficiently visualize labeled cells by fluorescence microscopy, the cytotoxicity of QDot500 and QDot600 was investigated (Fig. 3A, Supporting Fig. S5). The toxicity levels of both types of QDots were quite similar, where significant toxicity was observed at concentrations of $50 \mathrm{nM}$ for C17.2 and HUVEC cells and at $40 \mathrm{nM}$ for PC12 cells. The seemingly higher level of toxicity of the QDots for PC12 cells seems contradictory to the observed cellular uptake levels, which are lowest for the PC12 cells. As NP toxicity is more closely correlated to the cellular NP level rather than the number of NPs originally administered to the cells [22], this would result in lower toxicity in PC12 cells. However, the PC12 cells, which are small cells with a limited cytoplasmic space, have been found to be very sensitive to cell stress [39]. Additionally, it has been shown that the size of the cell and its cytoplasm inversely correlates with the level of NP the cell can handle without any toxic effects [42]. As the toxic effects of QDots have been found to derive for a major part from the release of toxic $\mathrm{Cd} 2+$ or $\mathrm{Zn} 2+$ ions [23], the effect of possible QDot degradation on toxicity was also investigated using non-proliferating cell cultures (Fig. 3B). The data reveal no significant increase in toxicity up to 7 days for either type of QDot, which is in contrast to earlier studies with various types of core-shell QDots, where QDot toxicity increased with time $[19,22]$. These data again demonstrate the high stability of the QDots against intraendosomal degradation.

As NP toxicity, including QDot toxicity, has been shown to be related to the induction of reactive oxygen species (ROS), the cellular ROS levels were determined (Fig. 3C, Supporting Fig. S6). The data reveal a clear concentration-dependent induction of oxidative stress in all cell types, which is of a similar level for both types of QDots. The level of oxidative stress correlates with the degree of toxicity, suggesting that oxidative stress may be an important cause of the observed cytotoxicity. However, the level of oxidative stress does not correlate with the number of cell-associated QDots. PC12 cells display the highest level of toxicity and oxidative stress, although these cells contain the 
lowest number of cell-associated QDots. This apparent discrepancy corresponds to differences in the sensitivity of different cells to foreign material as well as with differences in their antioxidative capacity [43]. This has been observed in different studies [19,43] and highlights the need for the use of multiple cell types when assessing particle toxicity in order to obtain a more general overview. To verify the correlation between oxidative stress and cytotoxicity, cells were co-treated with $\mathrm{N}$ acetylcystein (NAC), an FDA-approved free radical scavenger, which reduced cellular ROS to near control levels (Supporting Fig. S6). The addition of NAC also reduced cytotoxicity to a large extent (Fig. 3D, Supporting Fig. S5), indicating the importance of ROS in the observed cytotoxicity of the QDots, which is in line with many other studies [44].

\subsection{Effect of QDots on cell functionality}

Many different types of NPs have also been found to affect cellular morphology [14,45], for instance by deregulation of the cytoskeletal architecture of the cells [46]. These effects have been found to occur at conditions at which the NPs did not cause any acute cytotoxicity [47]. As the actin and tubulin cytoskeleton are very important mediators in various cell signaling pathways [48], and as for bio-imaging purposes it is essential that cells maintain their original morphology, the effect of QDot500 and QDot600 on HUVEC morphology was investigated. Fig. 4A shows a clear distribution of the cell areas of the HUVEC cells exposed to the QDots at concentrations up to $30 \mathrm{nM}$ (below the threshold for any cytotoxicity or oxidative stress to occur as high levels of cell death or ROS would influence the outcome of these results) calculated for various images of treated cells stained for F-actin and $\alpha$-tubulin (Fig. 4B). The data clearly show that both types of QDots affect cell morphology at concentrations of $20 \mathrm{nM}$, again revealing the great similarity between both types of QDots in terms of their effect on cultured cells.

As NPs can interact with cultured cells through a wide variety of different mechanisms [49], it is important to verify the functionality of the cells after cell labeling. For C17.2 and PC12 cells, the functionality of the cells was not investigated by their cellular spreading, as these cells are more rounded and have a less well-defined cytoskeleton, which hardens any straightforward analysis. Therefore, functionality of C17.2 and PC12 cells was investigated by means of cellular differentiation and neurite outgrowth assays, respectively. Fig. 4C shows that C17.2 differentiation is slightly affected by QDot exposure, where significant effects are observed at concentrations of $20 \mathrm{nM}$ or above. Fig. 4D shows that neurite outgrowth of PC12 cells is significantly impeded at concentrations of $15 \mathrm{nM}$ or higher. The higher sensitivity of the PC12 cells compared to C17.2 or HUVEC cells is somewhat surprising, given the lower cellular uptake and lower ROS levels in the PC12 cells. This difference may be explained by differences in the cell types, where PC12 cells themselves are inherently smaller than the C17.2 or HUVEC cells and can thus maximally accommodate a lower number of NPs in the restricted space of their cytoplasm, which may result in a higher sensitivity of 
the smaller (PC12) cells [42]. Alternatively, the higher sensitivity of the PC12 cells can also be due to the fact that the natural defense mechanisms (e.g. antioxidants) in the PC12 cells may be lower, which would make the cells more sensitive to (oxidative) stress [43]. A final reason for this difference may lie in the assay itself. Cell morphology assays are inherently not very sensitive due to the broad distribution of cell areas. Cell differentiation assays such as performed for the C17.2 cells are also less sensitive as they take several days to perform, during which time the number of QDots per cell may have diluted. Furthermore, during the differentiation process, the cells undergo high levels of stress and the assay is accompanied by significant cell death [50]. Due to this, the cells that contain the highest number of NPs might die and the assay itself will mostly represent cells with lower numbers of NPs and may thus not be a completely accurate overview of NP toxicity. The PC12 neurite outgrowth assay has however been shown to be a very sensitive assay that can be rapidly performed with minimal stress on the cultured cells, thus being optimally suited for determining NP toxicity [51].

\subsection{Usefulness of QDots for cell labeling}

Based on the different experiments performed, the various assays showed that both types of QDots did not affect any cellular parameter at concentrations up to $10 \mathrm{nM}$. Therefore, the safe concentration for cell labeling was determined at $10 \mathrm{nM}$. To determine how useful the gradient alloy QDots are for cell labeling, their functionality must however be assessed at their non-toxic concentration. Therefore, the QDots were used to label the different cell types at $10 \mathrm{nM}$, after which the cells were kept in culture and imaged every 2 days to see how long the cells can be efficiently visualized. Fig. 5 shows that the QDots can be used to monitor every type of cell for up to at least 6 cell doublings before the number of QDots is diluted too much due to continuous cell division.

The QDots appear to be quite well suited for cell labeling experiments. Previous studies on core-shell QDots investigated under identical conditions resulted in toxic levels of 0.5-2 $\mathrm{nM}$ [19,22], which is approximately 5-fold lower than the level found for the gradient alloy QDots examined in the current study. This value is however quite insignificant as determining the concentration of NPs is technically very challenging [13]. A direct comparison of NP toxicity based on no-observed adverse effect levels (NOAELs) is therefore often quite difficult and prone to errors based on incorrect determination of the original particle concentration. Determination of particle functionality, such as the visualization of labeled cells by fluorescence microscopy is therefore a far better alternative. When comparing the efficiency of cell visualization, the gradient alloy QDots allow 50\% of the cell population to be seen after 6 cell doublings, where the core-shell QDots studied previously under identical conditions led to cell visualization for 4 doublings [19,22]. This suggests that the gradient alloy QDots are approximately 1.5-fold more efficiently for cell visualization than the core-shell QDots. This value is a lot lower than the 5-fold increase in efficiency based on NOAELs and might be due to various factors, such as inaccurate determination of QDot concentrations or differences in the surface chemistry of the 
QDots, where the short ligands used in the current study are known to result in less colloidal stability and hence less cellular uptake than more stable polymer-coated QDots [52]. In conclusion, the gradient alloy QDots do have a big potential for cell labeling studies, where their high brightness and chemical stability result in good probes for long term cell visualization. Further improvements lie in the surface chemistry of the QDots, where polymer or silica coatings may enhance colloidal and chemical stability and improve cellular uptake levels.

The present study has, in contrast to most nanotoxicity studies, addressed the actual functionality of the QDots at their non-toxic concentration rather than providing a "safe concentration". Many studies also focus on the mechanisms of QDot toxicity and therefore employ CdTe core-only QDots $[20,23,46,53]$, which are known to be much more toxic than the core-shell QDots. The current study focuses on QDots that have a great potential for biomedical use and seeks to assess the potential of these QDots for their intended purpose of fluorescence-based imaging at non-toxic conditions. Using the multiparametric methodology, the values obtained can then also immediately be compared to those obtained for other types of QDots that were studied under identical conditions [19,22]. Placing the currently studied QDots in relation to various other types of QDots in terms of their functionality greatly enhances the power of the current study in contrast to most stand-alone studies, where it is almost impossible to make a proper comparison between results due to differences in the study designs and lack of appropriate standards being used.

\subsection{The effect of similar sizes with regard to intracellular QDot localization}

Common core-only or coreeshell QDots can span the entire visible range by altering the size of the QDot cores. QDots of different sizes have been found to reach different intracellular localizations [53]. Gradient alloy QDots can span the entire visible range while having identical sizes. As the particles studied in the present work also have identical surface chemistry, their intracellular localization may be more similar. This was tested by co-exposing cultured cells to QDot500 and QDot600 particles at $10 \mathrm{nM}$ concentrations for $24 \mathrm{~h}$ (Fig. 6A), after which their intracellular localization was compared to cells exposed to MPA-coated CdTe QDots of 2.6 and $6.4 \mathrm{~nm}$ diameter (510 and $630 \mathrm{~nm}$ wavelength, respectively) (Fig. 6B). These data clearly show a uniform labeling of the cells by the gradient alloy QDots, where colocalization of the two types of QDots reaches $91 \pm 7 \%$. For the CdTe QDots that differ widely in size $(2.6 \mathrm{e} 6.4 \mathrm{~nm})$ but have identical surface coating, their intracellular localization patterns differ more, where colocalization is limited to $58 \pm 9 \%$. The differences in intracellular localization of the QDots depending on their size is in line with earlier studies, where smaller QDots were found to reach different intracellular organelles than bigger sized QDots [53].

This size-dependent intracellular localization may be useful in certain conditions, if specific organelles would be targeted that cannot be reached by larger sized particles. However, to date, our understanding of the link between NP size and their precise intracellular location is still unclear and 
more research would be needed in order to be able to fully exploit this property for cell labeling or delivery purposes. Therefore, current methods to enable intracellular targeting are mostly limited to the use of surface attached ligands to specific cellular markers [54]. However, considering the difference in intracellular distribution of the QDots depending on their size, the addition of certain targeting moieties does not always give the expected results as these particles undergo different endocytic routes and particles of certain sizes may be hindered to reach their target site. Alternatively, as the gradient alloy QDots can be made of the same size and reach the same intracellular locations, this presents particularly useful properties with regard to their potential targeting capacities. As all QDots, regardless of their emission maxima, have the same size and surface characteristics, they will have the same intracellular distribution. This will make targeting methods by altering their surface coating more straightforward. 


\section{Conclusions}

The present work shows that GA-QDots have a high brightness and high chemical stability that appears to protect them for environmentally induced degradation. These parameters result in relatively low levels of cytotoxicity compared to common core-shell QDots. The differences in chemical composition of the QDot cores appear to only have a minimal effect on the extent and nature of cellQDot interaction, where cellular uptake levels and toxicity levels were similar for the two types of GA-QDots for all parameters studied. The data show that the GA-QDots are less toxic than common core-shell QDots, and affect cells through induction of ROS, disturbance of cell cytoskeletal architecture and impeded neuronal differentiation. However, when also assessing particle functionality, the difference between GA-QDots and common core-shell QDots is not as high as would be expected from comparing non-toxic concentrations. This difference stipulates the importance of using particle functionality for comparative purposes as particle concentrations are less appropriate, given that they do not take into account multiple important factors such as differences in particle brightness, differences in cellular uptake levels or possible errors in determining nanoparticle stock concentrations. The identical size of the GA-QDots furthermore offers a lot of interesting opportunities for cell labeling applications, such as drug delivery. Where common QDots with different emission maxima and thus different sizes result in quite different intracellular particle distributions, this is not the case for the GA-QDots, which given their identical sizes result in highly similar intracellular particle distributions. This finding offers a lot of potential for optimizing drug delivery studies, where the impact of the size of the delivery vehicle on its intracellular location is very important and should be optimized in terms of the final application of the nanomaterials. 


\section{Acknowledgments}

SJS is a post-doctoral fellow from the FWO-Flanders. Dr. Tangi Aubert and Prof. Zeger Hens (Ghent University, Belgium) are gratefully acknowledged for their help in elemental analysis and TEM.

\section{Appendix A. Supporting information}

Supporting information related to this article can be found at http://dx.doi.org/10.1016/j.biomaterials.2014.05.023. 


\section{References}

[1] Michalet X, Pinaud FF, Bentolila LA, Tsay JM, Doose S, Li JJ, et al. Quantum dots for live cells, in vivo imaging, and diagnostics. Science 2005;307:538-44.

[2] Medintz IL, Uyeda HT, Goldman ER, Mattoussi H. Quantum dot bioconjugates for imaging, labelling and sensing. Nat Mater 2005;4:435-46.

[3] Smith AM, Duan HW, Mohs AM, Nie SM. Bioconjugated quantum dots for in vivo molecular and cellular imaging. Adv Drug Deliv Rev 2008;60:1226-40.

[4] Chan WCW, Maxwell DJ, Gao XH, Bailey RE, Han MY, Nie SM. Luminescent quantum dots for multiplexed biological detection and imaging. Curr Opin Biotechol 2002;13:40-6.

[5] Peng CW, Tian Q, Yang GF, Fang M, Zhang ZL, Peng J, et al. Quantum-dots based simultaneous detection of multiple biomarkers of tumor stromal features to predict clinical outcomes in gastric cancer. Biomaterials 2012;33:5742-52.

[6] Chen C, Sun SR, Gong YP, Qi CB, Peng CW, Yang XQ, et al. Quantum dots-based molecular classification of breast cancer by quantitative spectroanalysis of hormone receptors and HER2. Biomaterials 2011;32:7592-9.

[7] Courty S, Luccardini C, Bellaiche Y, Cappello G, Dahan M. Tracking individual kinesin motors in living cells using single quantum-dot imaging. Nano Lett 2006;6:1491-5.

[8] Dahan M, Levi S, Luccardini C, Rostaing P, Riveau B, Triller A. Diffusion dynamics of glycine receptors revealed by single-quantum dot tracking. Science 2003;302:442-5.

[9] Ludolph B, Malik MA, O'Brien P, Revaprasadu N. Novel single molecule precursor routes for the direct synthesis of highly monodispersed quantum dots of cadmium or zinc sulfide or selenide. Chem Commun; 1998:1849-50.

[10] Walling MA, Novak JA, Shepard JRE. Quantum dots for live cell and in vivo imaging. Int J Mol Sci 2009;10:441-91.

[11] Rzigalinski BA, Strobl JS. Cadmium-containing nanoparticles: perspectives on pharmacology and toxicology of quantum dots. Toxicol Appl Pharmacol 2009;238:280-8.

[12] Soenen SJ, Demeester J, De Smedt SC, Braeckmans K. Turning a frown upside down: exploiting nanoparticle toxicity for anticancer therapy. Nano Today 2013;8:121-5. 
[13] Rivera-Gil P, De Aberasturi DJ, Wulf V, Pelaz B, Del Pino P, Zhao YY, et al. The challenge to relate the physicochemical properties of colloidal nanoparticles to their cytotoxicity. Acc Chem Res 2013;46:743-9.

[14] Soenen SJ, Rivera-Gil P, Montenegro JM, Parak WJ, De Smedt SC, Braeckmans K. Cellular toxicity of inorganic nanoparticles: common aspects and guidelines for improved nanotoxicity evaluation. Nano Today 2011;6:446-65.

[15] Tsoi KM, Dai Q, Alman BA, Chan WC. Are quantum dots toxic? Exploring the discrepancy between cell culture and animal studies. Acc Chem Res 2012;46:662-71.

[16] Joris F, Manshian BB, Peynshaert K, De Smedt SC, Braeckmans K, Soenen SJ. Assessing nanoparticle toxicity in cell-based assays: influence of cell culture parameters and optimized models for bridging the in vitroein vivo gap. Chem Soc Rev 2013;42:8339-59.

[17] Ye L, Yong K-T, Liu L, Roy I, Hu R, Zhu J, et al. A pilot study in non-human primates shows no adverse response to intravenous injection of quantum dots. Nat Nanotechnol 2012;7:453-8.

[18] Sealy C. Primates show mixed response to quantum dots. Nano Today 2012;7:223-4.

[19] Soenen SJ, Demeester J, De Smedt SC, Braeckmans K. The cytotoxic effects of polymer-coated quantum dots and restrictions for live cell applications. Biomaterials 2012;33:4882-8.

[20] Cho SJ, Maysinger D, Jain M, Roder B, Hackbarth S, Winnik FM. Long-term exposure to CdTe quantum dots causes functional impairments in live cells. Langmuir 2007;23:1974-80.

[21] Gagne F, Maysinger D, Andre C, Blaise C. Cytotoxicity of aged cadmium telluride quantum dots to rainbow trout hepatocytes. Nanotoxicology 2008;2:113-20.

[22] Soenen SJ, Montenegro JM, Abdelmonem AM, Manshian BB, Doak SH, Parak WJ, et al. The effect of nanoparticle degradation on poly(methacrylic acid)-coated quantum dot toxicity: the importance of particle functionality assessment in toxicology. Acta Biomater 2014;10:732-41.

[23] Su Y, Hu M, Fan C, He Y, Li Q, Li W, et al. The cytotoxicity of CdTe quantum dots and the relative contributions from released cadmium ions and nanoparticle properties. Biomaterials 2010;31:4829-34.

[24] Liu L, Hu R, Law WC, Roy I, Zhu J, Ye L, et al. Optimizing the synthesis of red and nearinfrared CuInS2 and AgInS2 semiconductor nanocrystals for bioimaging. Analyst 2013;138:6144-53. 
[25] Li L, Daou TJ, Texier I, Tran TKC, Nguyen QL, Reiss P. Highly luminescent CuInS2/ZnS core/shell nanocrystals: cadmium-free quantum dots for in vivo imaging. Chem Mater 2009;21:24229.

[26] Hu XG, Gao XH. Silica-polymer dual layer-encapsulated quantum dots with remarkable stability. ACS Nano 2010;4:6080-6.

[27] Yuan ZM, Wang JR, Yang P. Highly luminescent CdTe/CdS/ZnO core/shell/shell quantum dots fabricated using an aqueous strategy. Luminescence 2013;28:169-75.

[28] Maikov GI, Vaxenburg R, Sashchiuk A, Lifshitz E. Composition-tunable optical properties of colloidal IVeVI quantum dots, composed of core/shell heterostructures with alloy components. ACS Nano 2010;4:6547-56.

[29] Zhang W, Zhang H, Feng Y, Zhong X. Scalable single-step noninjection synthesis of high-quality core/shell quantum dots with emission tunable from violet to near infrared. ACS Nano 2012;6:1106673.

[30] Bailey RE, Nie SM. Alloyed semiconductor quantum dots: tuning the optical properties without changing the particle size. J Am Chem Soc 2003;125:7100-6.

[31] Cho J, Jung YK, Lee JK. Kinetic studies on the formation of various II-VI semiconductor nanocrystals and synthesis of gradient alloy quantum dots emitting in the entire visible range. J Mater Chem 2012;22:10827-33.

[32] Wang XY, Ren XF, Kahen K, Hahn MA, Rajeswaran M, Maccagnano-Zacher S, et al. Nonblinking semiconductor nanocrystals. Nature 2009;459:686-9.

[33] Smith AM, Nie SM. Next-generation quantum dots. Nat Biotechnol 2009;27:732-3.

[34] Ruan G, Agrawal A, Marcus AI, Nie S. Imaging and tracking of tat peptide-conjugated quantum dots in living cells: new insights into nanoparticle uptake, intracellular transport, and vesicle shedding. J Am Chem Soc 2007;129:14759-66.

[35] Zhu ZJ, Yeh YC, Tang R, Yan B, Tamayo J, Vachet RW, et al. Stability of quantum dots in live cells. Nat Chem 2011;3:963-8.

[36] Chang E, Thekkek N, Yu WW, Colvin VL, Drezek R. Evaluation of quantum dot cytotoxicity based on intracellular uptake. Small 2006;2:1412-7.

[37] Chen N, He Y, Su Y, Li X, Huang Q, Wang H, et al. The cytotoxicity of cadmium-based quantum dots. Biomaterials 2012;33:1238-44. 
[38] Soenen SJ, Himmelreich U, Nuytten N, De Cuyper M. Cytotoxic effects of iron oxide nanoparticles and implications for safety in cell labelling. Biomaterials 2011;32:195-205.

[39] Soenen SJ, Manshian B, Montenegro JM, Amin F, Meermann B, Thiron T, et al. Cytotoxic effects of gold nanoparticles: a multiparametric study. ACS Nano 2012;6:5767-83.

[40] Ju L, Zhang G, Zhang C, Sun L, Jiang Y, Yan C, et al. Quantum dot-related genotoxicity perturbation can be attenuated by PEG encapsulation. Mutat Res 2013;753:54-64.

[41] Mittal R, Bruchez MP. Calibration of flow cytometry for quantitative quantum dot measurements. Curr Protoc Cytom; 2009 [Chapter 6: Unit6 26].

[42] Ketkar-Atre A, Struys T, Soenen SJ, Lambrichts I, Verfaillie CM, De Cuyper M, et al. Variability in contrast agent uptake by different but similar stem cell types. Int J Nanomed 2013;8:4577-91.

[43] Diaz B, Sanchez-Espinel C, Arruebo M, Faro J, de Miguel E, Magadan S, et al. Assessing methods for blood cell cytotoxic responses to inorganic nanoparticles and nanoparticle aggregates. Small 2008;4:2025-34.

[44] Nguyen KC, Willmore WG, Tayabali AF. Cadmium telluride quantum dots cause oxidative stress leading to extrinsic and intrinsic apoptosis in hepatocellular carcinoma HepG2 cells. Toxicology 2013;306:114-23.

[45] Tay CY, Cai PQ, Setyawati MI, Fang WR, Tan LP, Hong CHL, et al. Nanoparticles strengthen intracellular tension and retard cellular migration. Nano Lett 2014;14:83-8.

[46] Okoturo-Evans O, Dybowska A, Valsami-Jones E, Cupitt J, Gierula M, Boobis AR, et al. Elucidation of toxicity pathways in lung epithelial cells induced by silicon dioxide nanoparticles. PLoS One 2013;8:e72363.

[47] Soenen SJ, Nuytten N, De Meyer SF, De Smedt SC, De Cuyper M. High intracellular iron oxide nanoparticle concentrations affect cellular cytoskeleton and focal adhesion kinase-mediated signaling. Small 2010;6:832-42.

[48] Ridley AJ, Schwartz MA, Burridge K, Firtel RA, Ginsberg MH, Borisy G, et al. Cell migration: integrating signals from front to back. Science 2003;302:1704-9.

[49] Wu YL, Putcha N, Ng KW, Leong DT, Lim CT, Loo SCJ, et al. Biophysical responses upon the interaction of nanomaterials with cellular interfaces. Acc Chem Res 2013;46:782-91.

[50] Soenen SJ, De Cuyper M. Assessing iron oxide nanoparticle toxicity in vitro: current status and future prospects. Nanomedicine Lond 2010;5:1261-75. 
[51] Pisanic 2nd TR, Blackwell JD, Shubayev VI, Finones RR, Jin S. Nanotoxicity of iron oxide nanoparticle internalization in growing neurons. Biomaterials 2007;28:2572-81.

[52] Kirchner C, Liedl T, Kudera S, Pellegrino T, Munoz Javier A, Gaub HE, et al. Cytotoxicity of colloidal CdSe and CdSe/ZnS nanoparticles. Nano Lett 2005;5:331-8.

[53] Lovric J, Bazzi HS, Cuie Y, Fortin GR, Winnik FM, Maysinger D. Differences in subcellular distribution and toxicity of green and red emitting CdTe quantum dots. J Mol Med Berl 2005;83:37785.

[54] Zhang MZ, Yu Y, Yu RN, Wan M, Zhang RY, Zhao YD. Tracking the downregulation of folate receptor-alpha in cancer cells through target specific delivery of quantum dots coupled with antisense oligonucleotide and targeted peptide. Small 2013;9:4183-93. 
Table 1

Elemental composition of QDot500 and QDot600.

$\begin{array}{lllll} & \mathrm{Zn} & \mathrm{Se} & \mathrm{Cd} & \mathrm{S} \\ \text { QDot500 } & 68 \pm 6 & 1 \pm 1 \% & 1 \pm 1 \% & 30 \pm 6 \% \\ \text { QDot600 } & 34 \pm 3 \% & 6 \pm 1 \% & 19 \pm 1 \% & 41 \pm 4 \%\end{array}$




\section{Figure legends}

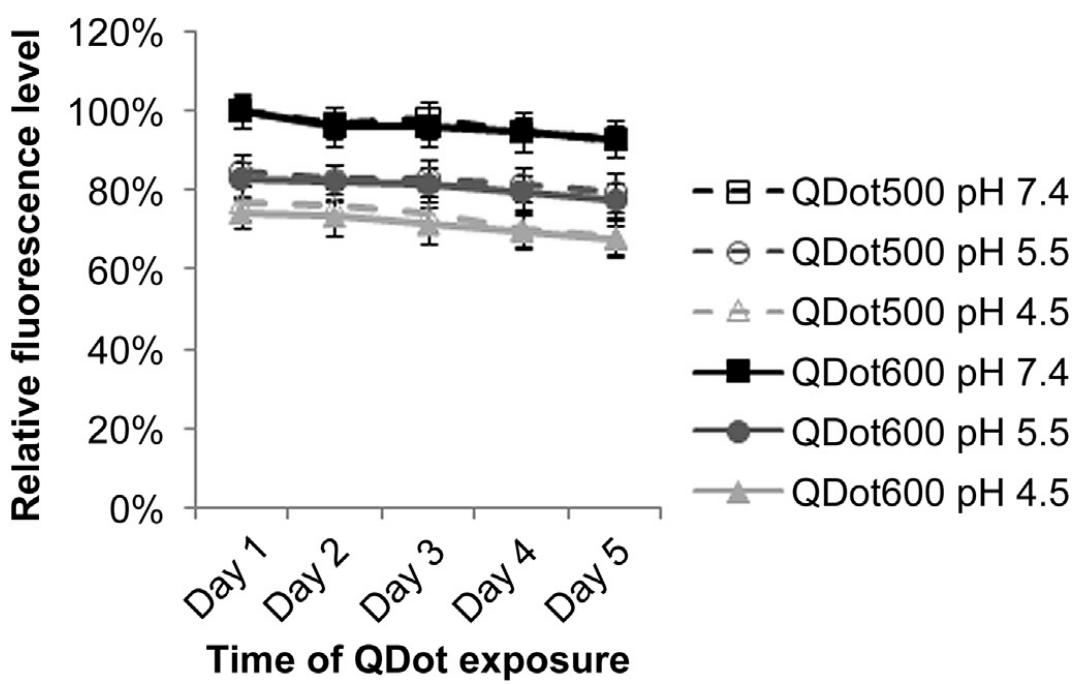

Fig. 1. The fluorescence intensity levels of QDot500 and QDot600 exposed to buffer systems of different pH values, being 7.4 (cytoplasmic); 5.5 (late endosomes) or 4.5 (lysosomes) for up to 5 days. Fluorescence intensity levels were measured every day. Data are presented as values relative to the fluorescence intensity of QDots exposed to $\mathrm{pH} 7.4$ and measured immediately (day 0 1/4 100\%). Data are expressed as mean $\pm \operatorname{SEM}(n=3)$. 


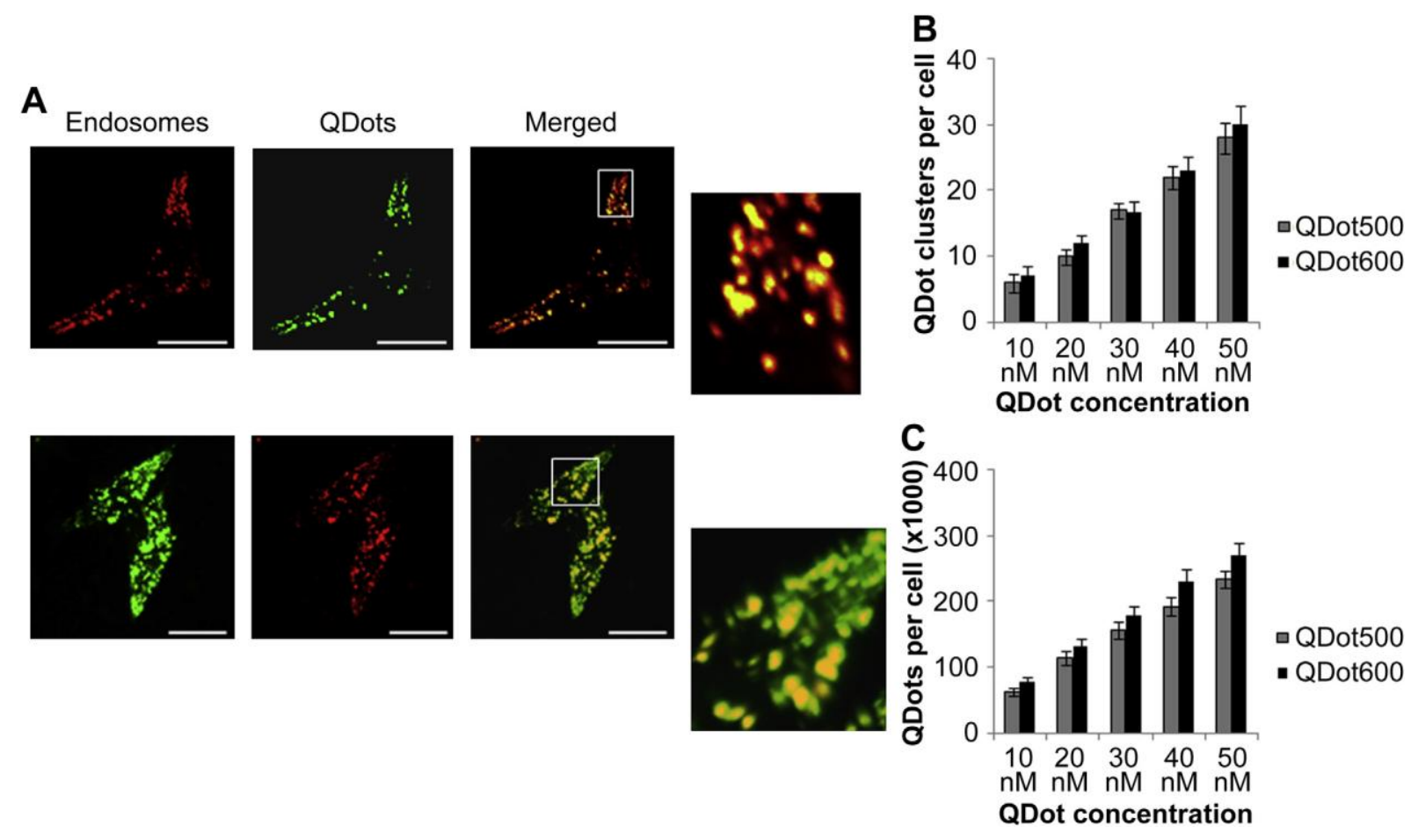

Fig. 2. Cellular uptake of QDots. (A) Representative confocal images of HUVEC cells transiently expressing fluorescently tagged Lamp-1 (marker for late endosomes and lysosomes) incubated with 30 nM QDot500 (top row; green channel) or QDot600 (bottom row; red channel) for $24 \mathrm{~h}$. Cells exposed to QDot500 are tagged with Lamp-1-RFP (red channel); cells exposed to QDot600 are tagged with Lamp-1-GFP. A merged image of both the QDots and the fluorescently tagged endosomes is shown in the right column. The area indicated by the white rectangle in the merged images is shown in high resolution on the right. Scale bars: $40 \mu \mathrm{m}$. (B) The number of QDot clusters per cell as a function of the QDot concentration for both QDot500 (light gray) and QDot600 (dark gray) as quantified from 20 microscopy images. (C) The total number of QDots per HUVEC cell as a function of the QDot concentration for both QDot500 (light gray) and QDot600 (dark gray) as quantified by measuring total fluorescence intensity levels. Data are shown as mean $\pm \operatorname{SEM}(n=4)$. 

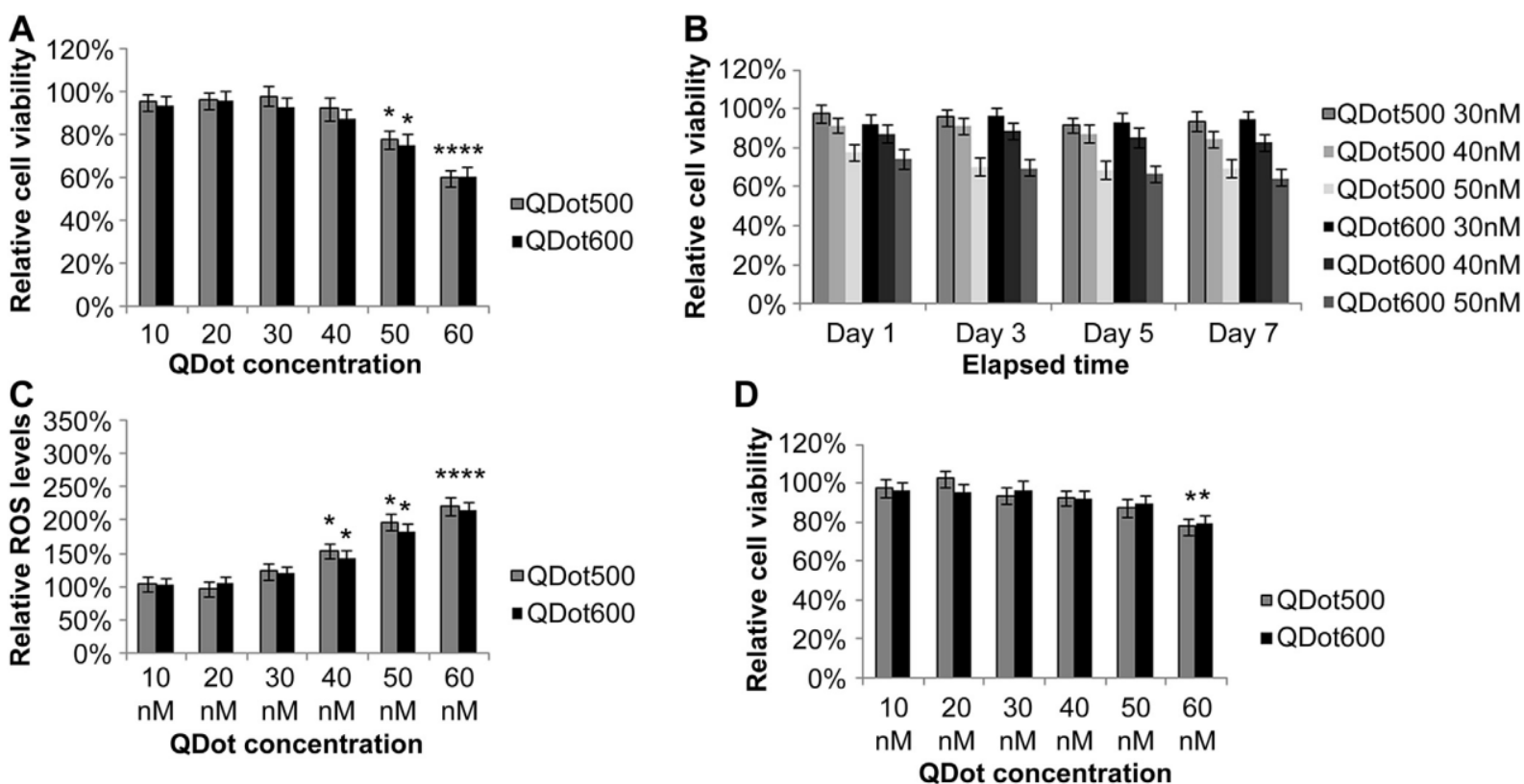

Fig. 3. (A) Viability of HUVEC cells as a function of QDot concentration (ranging from 10 to $60 \mathrm{nM}$ ) for both QDot500 (light gray) and QDot600 (dark gray) after 24 h incubation. (B) Viability of nonproliferating HUVEC cells as a function of both QDot concentrations (30, 40 and $50 \mathrm{nM}$ ) and time (17 days post-QDot incubation). (C) ROS levels of HUVEC cells exposed to varying concentrations (10$60 \mathrm{nM}$ ) of QDot500 (light gray) or QDot600 (dark gray). (D) Viability of HUVEC cells as a function of QDot concentration (ranging from 10 to $60 \mathrm{nM}$ ) for both QDot500 (light gray) and QDot600 (dark gray) after $24 \mathrm{~h}$ incubation in the presence of $5 \mathrm{mM}$ NAC, a free radical scavenger. Data are represented as mean \pm SEM $(n=4)$ and expressed as relative to untreated control cells $(100 \%)$ or for figure B as treated cells at day $0(100 \%)$. The degree of significance is indicated when appropriate $(*$ : $\mathrm{p}<0.05 ; * *: \mathrm{p}<0.01 ; * * * \mathrm{p}<0.001)$. 


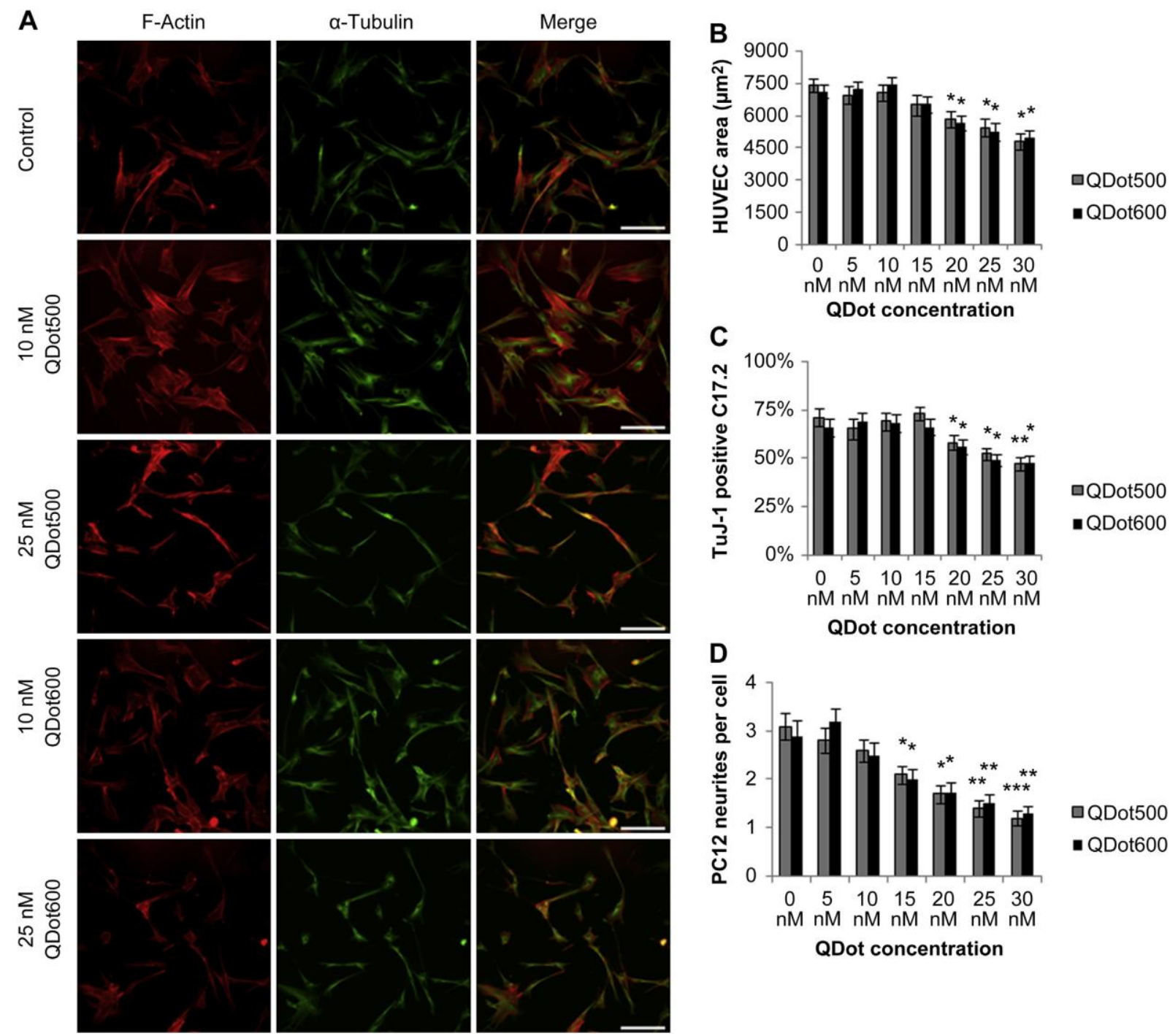

Fig. 4. (A) Representative fluorescence images of HUVEC cells either untreated (top row) or exposed to $10 \mathrm{nM}$ QDot500 (second row), $25 \mathrm{nM}$ QDot500 (third row), $10 \mathrm{nM}$ QDot600 (fourth row) or $25 \mathrm{nM}$ QDot600 (bottom row) for $24 \mathrm{~h}$. Cells have been stained for F-actin (red, left column) and $\alpha$-tubulin (green, middle column). The right column shows a merged image of both the green and red channel. Scale bars: $150 \mu \mathrm{m}$. (B) The average cell area of HUVEC cells as a function of QDot concentration (ranging from 0 to $30 \mathrm{nM}$ ) for both QDot500 (light gray) and QDot600 (dark gray) after $24 \mathrm{~h}$ incubation. (C) The level of C17.2 cells that differentiated into full neurons upon 1 week exposure to neuronal induction media as a function of QDot concentration (ranging from 0 to $30 \mathrm{nM}$ ) for both QDot500 (light gray) and QDot600 (dark gray) after $24 \mathrm{~h}$ incubation. (D) The level of PC12 neurite outgrowth upon 2 days exposure to nerve growth factor as a function of QDot concentration (ranging from 0 to $30 \mathrm{nM}$ ) for both QDot500 (light gray) and QDot600 (dark gray) after $24 \mathrm{~h}$ incubation. (B-D) Data are expressed as mean \pm SEM $(n=4)$. The degree of significance is indicated when appropriate $(*: \mathrm{p}<0.05 ; * *: \mathrm{p}<0.01 ; * * *: \mathrm{p}<0.001)$. 

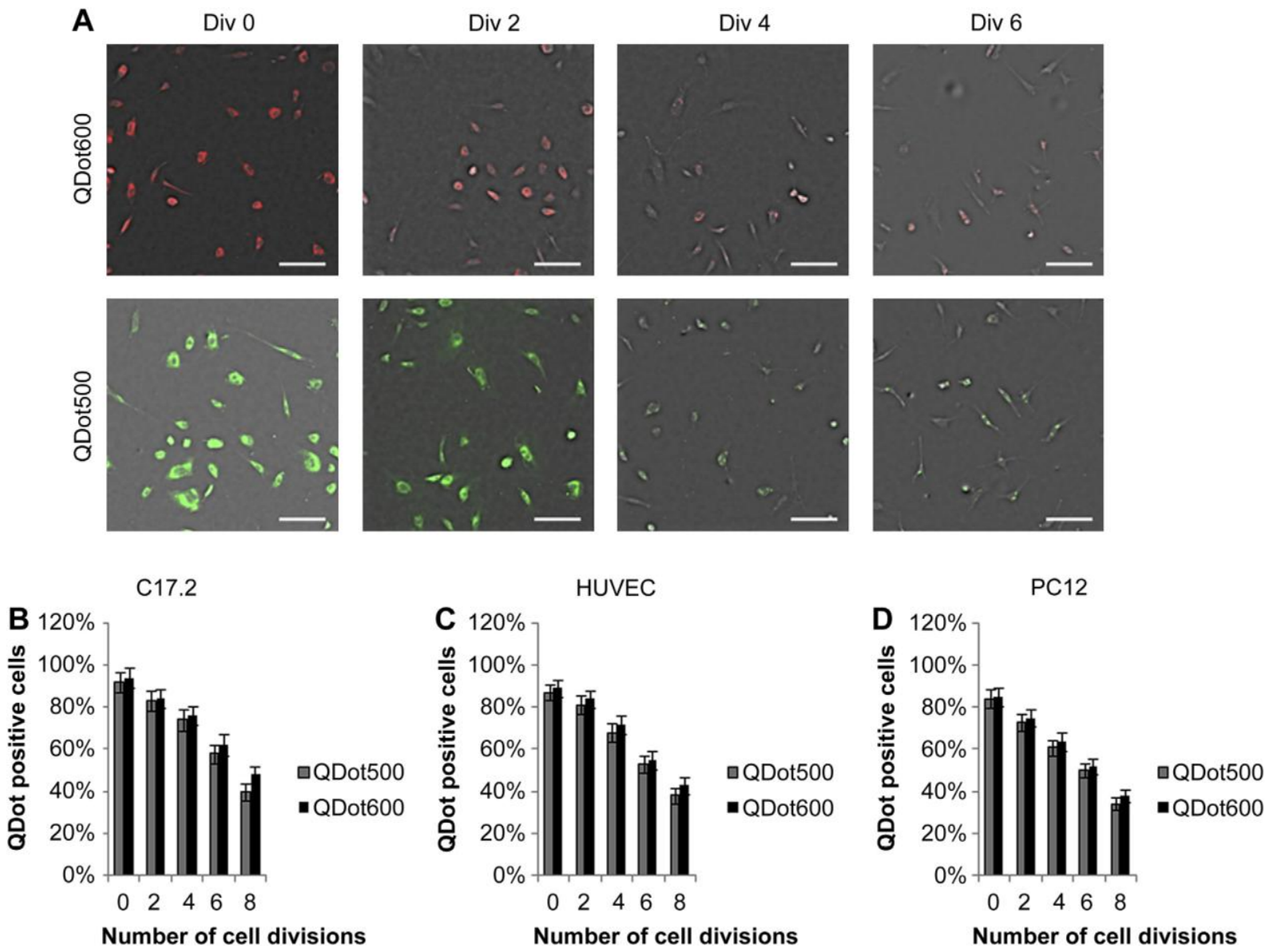

Fig. 5. (A) Representative fluorescence images of HUVEC cells exposed to non-cytotoxic concentrations of QDot600 (10 nM; top row) or QDot500 (10 nM; bottom row) for $24 \mathrm{~h}$, after which the cells were kept in culture. The images shown are taken immediately after QDot exposure (D0) or after 2 (D2), 4 (D4) or 6 (D6) average cell doubling times. The images are merged images of the fluorescent QDots (QDot500: green; QDot600: red) and phase contrast images of the cells. Scale bars: $150 \mu \mathrm{m}$. (B-D) The percentage of (B) C17.2, (C) HUVEC or (D) PC12 cells containing QDot500 (light gray) or QDot600 (dark gray) upon further culture of pre-incubated cells. The data are expressed as a function of the number of cell divisions the cells underwent post-incubation. Data are given as mean $\pm \operatorname{SEM}(\mathrm{n}=4)$. 

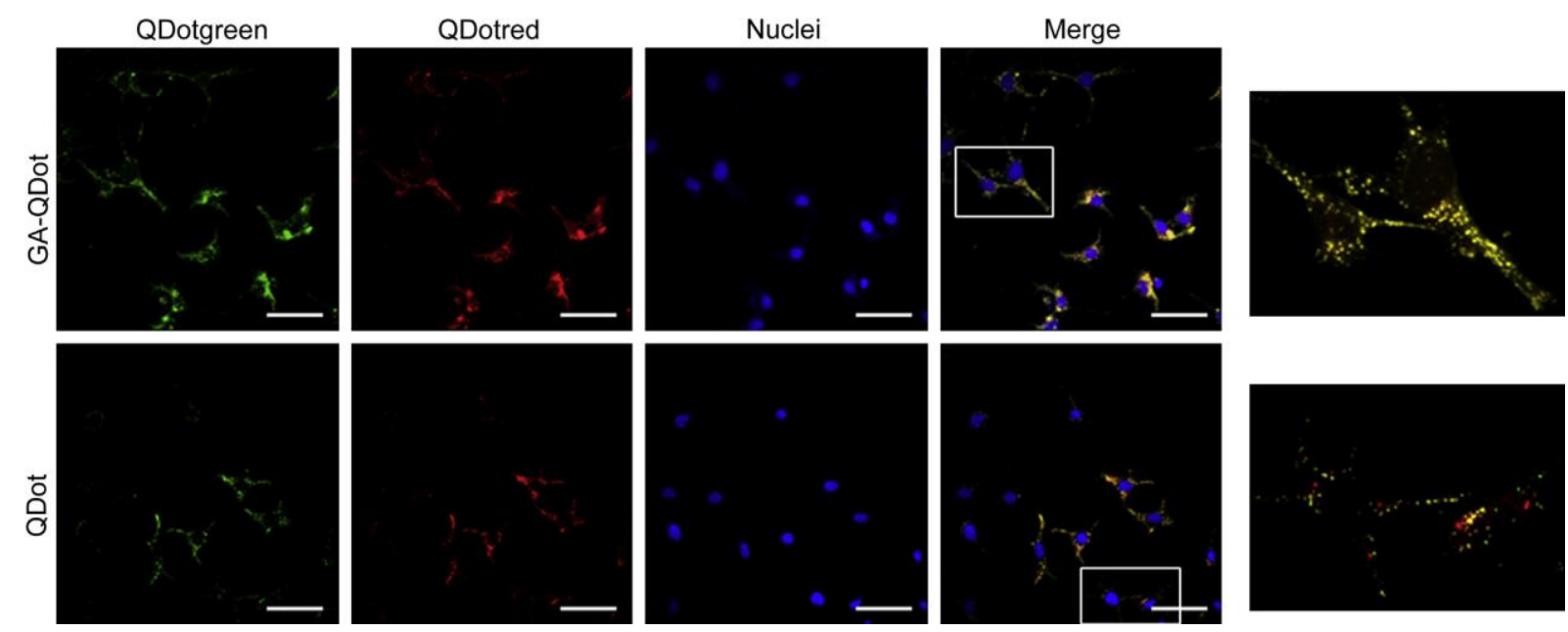

Fig. 6. Representative fluorescence images of HUVEC cells co-incubated with green and red QDots for $24 \mathrm{~h}$, either GA-QDots of similar size (top row) or MPA-coated CdTe QDots of different sizes (bottom row). Cell nuclei were counterstained with DAPI (blue; third column). The fourth column shows merged images of the green, red and blue channel. The area indicated by the rectangle in the merged images is displayed as a magnified inset in the fifth column revealing high colocalization of the GA-QDots compared to lower levels of colocalization for the CdTe QDots. Scale bars: $150 \mu \mathrm{m}$. 